\title{
La construcción socioimaginaria de la protesta social en el discurso de la prensa latinoamericana: análisis de las editoriales de los diarios El Mercurio (Chile) y La Nación (Argentina) en el contexto de la crisis argentina de $2001^{*}$
}

\author{
Alberto Javier Mayorga Rojel ${ }^{\star \star}$ \\ Luis Nitrihual Valdebenito*** \\ Recibido: 2018-01-21. Enviado a pares: 2018-01 25. \\ Aprobado por pares: 2018-02-25. Aceptado: 2018-02-28 \\ https://doi.org/10.22395/angr.v17n33a8
}

\begin{abstract}
Resumen
El presente trabajo de investigación se propone como un espacio para la discusión teórica y EL análisis empírico en torno a la problemática del poder de los medios de comunicación en contextos de conflictividad social. De forma más precisa, este artículo tiene por finalidad analizar la construcción socioimaginaria de la protesta social en el discurso editorial producido por los diarios El Mercurio (Chile) y La Nación (Argentina) a partir de las movilizaciones populares que ocurrieron durante diciembre de 2001 en el marco de la crisis económica en Argentina.
\end{abstract}

Para responder a nuestra pregunta de investigación y cumplir con el objetivo del estudio, se utilizó un modelo de análisis del discurso que fue aplicado a un corpus de 40 editoriales seleccionadas a partir de una muestra de 80 textos periodísticos publicados por los diarios El Mercurio y La Nación durante el periodo de tiempo que va desde el 1 diciembre de 2001 al 31 marzo de 2002.

Los principales hallazgos de la investigación obtenidos a partir del análisis de las editoriales publicadas por los diarios El Mercurio y La Nación en relación a las movilizaciones que ocurrieron en el contexto de la crisis económica en Argentina, nos permiten evidenciar que ambos medios de comunicación construyeron un imaginario de la protesta social sobre la base de un discurso centrado en cuatro categorías: civilización, barbarie, exclusión e inclusión. Por consiguiente, los diarios El Mercurio y La Nación no solo permitieron visibilizar en el espacio público los acontecimientos asociados a las protestas de diciembre de 2001, sino que, además, estos medios canalizaron, a través de sus discursos editoriales, las explicaciones en torno al conflicto, participaron del debate público respecto a las acciones colectivas de protesta, construyeron representaciones y articularon imaginarios acerca de los sucesos y de los actores sociales que participaron de las interacciones enmarcadas en el conflicto, y, por supuesto, asumieron una posición ideológica en su calidad de actores del sistema político respecto a la crisis económica y el conflicto social que se desarrolla en la sociedad argentina durante el 2001.

Palabras clave: prensa, imaginarios sociales, conflicto social, análisis del discurso, protesta social.

Artículo asociado a los proyectos de investigación Fondecyt 1180892, Fondecyt 1150666 y Fondecyt 1161253 financiados por la Comisión Nacional de Investigación Científica y Tecnológica del Estado de Chile y proyectos Diufro DI12-0038, Diufro DI18-0047 y Diufro DI18-0004, financiados por la Dirección de Investigación de la Universidad de La Frontera.

" Doctor en Comunicación por la Universidad Autónoma de Barcelona, director del Doctorado en Ciencias Sociales de la Universidad de La Frontera. alberto.mayorga@ufrontera.cl Orcid: https://orcid.org/0000-0002-7566-5045

... Doctor en Ciencias de la Información por la Universidad Complutense de Madrid, director del Centro de Investigación en Comunicación, Discurso y Poder de la Universidad de La Frontera. luis.nitrihual@ufrontera.cl Orcid: https://orcid. org/0000-0003-0013-1468 


\title{
The socioimaginary construction of social protest in the discourse of the latin american press: analysis of the editorials of El Mercurio (Chile) and la nación (Argentina) newspapers in the context of the argentine crisis of 2001
}

\begin{abstract}
The present research work is proposed as a space for theoretical discussion and empirical analysis around the problematic of the power of the media in contexts of social conflict. Explicitly, this paper aims to analyze the socioimaginary construction of social protest in the editorial discourse produced by El Mercurio (Chile) and La Nación (Argentina) newspapers, based on the popular demonstrations that took place during December 2001 in the framework of the economic crisis in Argentina.

To answer our research question and meet the goal of the study, we used a discourse analysis model that was applied to a corpus of 40 leading articles, selected from a sample of 80 journalistic texts published by El Mercurio and La Nación from December 1, 2001 to March 31, 2002.

The main findings of the research regarding the mobilizations that took place in the context of the economic crisis in Argentina allow us to show that both newspapers built an imaginary of social protest that was based on a discourse with four categories: Civilization, barbarism, exclusion, and inclusion. Therefore, El Mercurio and La Nación newspapers not only made visible in the public space the events associated with the protests of December 2001, but also, these media channeled, through their leading articles, explanations about the conflict. They also participated in the public debate regarding collective protest actions, constructed representations and imaginary articulations about the events and social actors that participated in the interactions framed in the conflict, and, of course, assumed an ideological position in their quality of actors of the political system regarding the economic crisis and the social conflict that happened in the Argentine society during 2001

Keywords: Press, social imaginaries, social conflict, discourse analysis, social protest.
\end{abstract}

\section{A construção socioimaginária do protesto social no discurso da imprensa latino-americana: análise dos editoriais dos jornais El Mercurio (Chile) e La Nación (Argentina) no contexto da crise argentina de 2001}

\begin{abstract}
Resumo
O presente trabalho de pesquisa propõe-se como um espaço para a discussão teórica e a análise empírica em torno da problemática do poder dos meios de comunicação em contextos de conflitividade social. De forma mais precisa, este artigo tem por finalidade analisar a construção socioimaginária do protesto social no discurso editorial produzido pelos jornais El Mercurio (Chile) e La Nación (Argentina) a partir das mobilizações populares que ocorreram durante dezembro de 2001 no âmbito da crise econômica na Argentina.

Para responder a nossa pergunta de pesquisa e cumprir o objetivo do estudo, utilizou-se um modelo de análise do discurso que foi aplicado a um corpus de 40 editoriais selecionados a partir de uma amostra de 80 textos jornalísticos publicados pelos jornais El Mercurio e La Nación durante o período que vai do dia 1 dezembro de 2001 a 31 março de 2002.

As principais descobertas da pesquisa obtidas a partir da análise dos editoriais publicados pelos jornais El Mercurio e La Nación com relação às mobilizações que ocorreram no contexto da crise econômica na Argentina, permitem-nos evidenciar que ambos os meios de comunicação construíram um imaginário do protesto social sobre a base de um discurso centrado em quatro categorias: civilização, barbárie, exclusão e inclusão. Portanto, os jornais El Mercurio e La Nación não apenas permitiram visibilizar no espaço público os acontecimentos associados aos protestos de dezembro de 2001, como também canalizaram, por meio de seus discursos editoriais, as explicações em torno do conflito, participaram do debate público com relação às ações coletivas de protesto, construíram representações e articularam imaginários sobre os acontecimentos e sobre os atores sociais que participaram das interações enquadradas no conflito, e que, desse modo, assumiram uma posição ideológica no papel de atores do sistema político com relação à crise econômica e o conflito social que se desenvolve na sociedade argentina durante 2001.

Palavras-chave: imprensa, imaginários sociais, conflito social, análise do discurso, protesto social.
\end{abstract}




\section{Introducción}

Al inicio del siglo XXI, los ciudadanos del mundo fuimos testigos a través de los medios de comunicación social de un conjunto de sucesos que se desarrollaron en varias ciudades de Argentina durante el mes de diciembre de 2001. En ese momento, se materializaba una crisis económica que impulsó a los argentinos a movilizarse en las calles y manifestar su descontento contra un modelo de desarrollo económico que no lograba dar respuesta a las necesidades de bienestar social y estabilidad política-económica.

Producto de esta situación de crisis, la sociedad civil argentina se manifestó en las calles por medio de acciones colectivas de protesta (Rossi, 2017; Rossi y Mauro, 2013; Ferrero, 2017; Auyero y Moran, 2007; Auyero, 2002, 2004 y 2006; Mauro y Natanson, 2006; Rodríguez, 2006) que se traducían en el corte de rutas (piquetes), el saqueo de locales comerciales, ocupación de plazas y edificios públicos, barricadas y enfrentamientos con la policía, y marchas masivas que desbordaban las principales arterias urbanas de las ciudades más importantes de Argentina, donde los sujetos sociales alzaban la voz para exclamar con fuerza: iQué se vayan todos!

Así entonces, esta conflictividad social que estalla en Argentina es el marco en el que se configuran los acontecimientos de movilización social que lograron desestabilizar al gobierno del presidente Fernando de la Rúa y, en consecuencia, a partir de esta conflictividad social que se traduce en las acciones colectivas de protesta más visibles de la crisis argentina, es cuando los medios de comunicación jugaron un papel fundamental en lo que respecta a su capacidad para producir un conjunto de discursos mediáticos que no responden a la objetividad de los sucesos tal como pueden ocurrir en el mundo (Artese, 2011; Î́nigo y Cotarelo, 2006; Neuhaus, 2002, Álvarez, Farré y Fernández, 2002; Sodré, 1998), sino más bien son los medios de comunicación los responsables de configurar una diversidad de mundos posibles (Farré, 2004; Curran, 2002; Rodrigo Alsina, 1996) sobre la base de un discurso capaz de ordenar, clasificar y organizar los hechos y conflictos que involucran a múltiples sujetos, a organizaciones e instituciones, dentro de un contexto referencial apropiado para promover un sentido socialmente aceptado por sus audiencias.

Incluso, podemos afirmar que, en contextos de conflicto social, los medios de comunicación tienen la capacidad de configurar las experiencias de las audiencias, lo que inevitablemente marca un quiebre en la frontera de las percepciones que el individuo tiene a partir de sus contactos cotidianos e inmediatos (Curran, 2002; Thompson, 1998; Sodré, 1998), para dar paso a una nueva realidad mediatizada por un medio de comunicación tradicional o digital (Brym, Godbout, Hoffbauer, Menard y Zhang, 2014) en la que las formas simbólicas que se acuñan en el conjunto de discursos mediáticos proporcionan las significaciones sociales que se transforman en el sustento para la producción o reproducción de un imaginario social, como sería en el caso de las protestas sociales acaecidas en el contexto de la crisis argentina de 2001.

Por ello, estimamos pertinente establecer que en los contextos de conflicto social altamente significativos e históricos, como es el caso de la crisis argentina de 2001, se hace 
necesario estudiar el discurso de la prensa como un camino apropiado para el análisis histórico de los imaginarios sociales de la protesta social, que nos permita profundizar en la discusión acerca del poder de los medios de comunicación en general, y de la prensa en particular, cuando se trata de describir el rol que ostentan los medios en el marco de los procesos sociales que experimentan los países afectados por dinámicas de violencia y caos producto de las crisis financieras que derivan en crisis políticas y sociales.

Además, resulta fundamental precisar que este trabajo se focaliza en el análisis del discurso editorial elaborado por la prensa chilena y argentina acerca de las protestas sociales que se gestaron durante diciembre de 2001 como consecuencia de la crisis económica argentina. En este sentido, el discurso editorial es fundamental para el análisis de la prensa en la medida en que este género periodístico corresponde a la práctica discursiva en la que una empresa periodística ejerce el derecho a expresar su posición respecto a los temas de interés nacional e internacional (Gronemeyer y Porath, 2017). Este tipo de discurso mediático construye la posición del medio y utiliza esta instancia de comunicación para definir su participación en el espacio público (Blanks, 2008) y, en definitiva, logra visibilizar la posición ideológica del medio respecto a los sucesos que acontecen en el mundo.

Por lo tanto, el discurso editorial es una característica central de la prensa que permite considerar a toda empresa periodística como un actor del sistema político (Borrat, 1989) que promueve determinados sistemas de ideas, define la forma como se estructura el conocimiento público, configura representaciones acerca de los sucesos de la realidad y participa de la organización en torno a la discusión pública que orienta los temas de la agenda (Lunt y Livingstone, 2013; Lukin, 2012; Simelio, 2009; Ferguson, 2007; Moreno Sardá, Simelio Solá, Rovetto y Buitrago, 2007; McCombs, 2006; Martín-Barbero, 2004; Curran, 2002; Hall, 1981 y 1998; Velázquez, 1992).

Por consiguiente, la importancia de este trabajo consiste en presentar un estudio del discurso editorial de los diarios El Mercurio (Chile) y La Nación (Argentina) sobre la base de cuatro componentes que forman parte de un modelo de análisis - figuras discursivas, recorridos narrativos, valores temáticos y elementos nucleares_ a fin de determinar la capacidad de estos medios de comunicación para establecer un imaginario de la protesta social en un contexto de conflicto articulado por la crisis económica de 2001 en Argentina. En esta medida, la pregunta que orienta este trabajo es la siguiente: ¿̇cómo se presenta el imaginario de la protesta social en el discurso editorial elaborado por los diarios El Mercurio de Chile y La Nación de Argentina a partir de los sucesos acaecidos durante diciembre de 2001 en el contexto de la crisis económica en Argentina?

Para los fines de esta investigación, los diarios fueron seleccionados al considerar su relevancia y legitimidad en lo que respecta a la conformación de la opinión pública en los territorios nacionales de ambos países y, a su vez, al destacar la importancia de estas empresas periodísticas en la influencia del quehacer político, económico y social de Chile y Argentina (Gronemeyer y Porath, 2017; Raimondo Anselmino, 2014; Mastrini y 
Becerra, 2006). Incluso, debemos destacar que los diarios El Mercurio de Chile y La Nación de Argentina son medios de comunicación que están relacionados con los grupos conservadores, católicos y empresariales más influyentes de ambos países, son dos de las empresas periodísticas con mayor antigüedad del cono sur y ambos diarios fueron los socios fundadores del consorcio exclusivo de periódicos independientes más importante en Latinoamérica como es el caso del Grupo de Diarios América.

Finalmente, es necesario presentar las secciones que definen el orden del texto. La primera sección corresponde al desarrollo teórico-conceptual respecto a las nociones de discurso de prensa e imaginarios sociales donde damos cuenta de algunos aportes académicos pertinentes que permiten dilucidar la importancia de ambas nociones para nuestra investigación. Luego, en una segunda sección se procede a describir los lineamientos metodológicos que sustentan este trabajo, particularmente lo referido a los fundamentos del análisis del discurso, la selección del corpus de análisis y aspectos centrales del modelo de análisis utilizado en esta investigación. La tercera sección se focaliza en la entrega de los resultados obtenidos a partir de la aplicación del modelo de análisis, aquí la presentación de los resultados se realiza bajo una modalidad de relato en torno a los hallazgos centrales de la investigación como modalidad que permite sintetizar la información para una mejor lectura y comprensión. Por último, la cuarta sección se centra en las consideraciones finales del trabajo donde esperamos dar cuenta de algunos lineamientos generales que permitan sostener la relevancia y pertinencia de la investigación sobre el discurso de la prensa que se genera a partir de sucesos que se enmarcan en contextos de conflicto social tanto en el pasado reciente, presente y futuro.

\section{Marco teórico-conceptual: discurso de prensa e imaginarios sociales}

En este apartado teórico-conceptual de nuestro trabajo nos parece necesario destacar que, a pesar del crisol informativo-comunicacional de carácter digital que se evidencia en la actualidad, aún son importantes los tradicionales medios de comunicación social en la configuración de un conjunto de realidades mediáticas que suministran una plétora informativa de aparente diversidad de imágenes y pluralidad de voces, lo cual permite que los medios de comunicación social mantengan una injerencia en la construcción socioimaginaria que las personas formulan a partir del consumo y la apropiación ${ }^{1}$ del mensaje (Thompson, 1998).

Así entonces, es factible evidenciar que los medios de comunicación social juegan un rol fundamental tanto en los procesos de configuración de las mentalidades (Baczko, 2005), en la producción de los discursos que conducen a los imaginarios sociales (Baeza, 2008 y 2015) y en los procesos de construcción social de la realidad (Berger y Luckman, 2001; Carter, 2013; Adoni y Mane, 1984), como, además, en la producción de universos

\footnotetext{
Utilizaremos el término apropiación en el sentido propuesto por John B. Thompson (1998): "consiste en asimilar el mensaje e incorporarlo a la propia vida, un proceso que algunas veces tiene lugar sin esfuerzos, y otras supone un esfuerzo consciente. Cuando nos apropiamos de un mensaje lo adaptamos a nuestras vidas y a los contextos en los que vivimos. Nos referimos a un conjunto de circunstancias que, en el caso de los productos mediáticos, difieren de las circunstancias en las que se produjo el mensaje" (p. 66).
} 
simbólicos, de consensos sociales, de legitimación de sedimentos ideológicos (Hall, 1981 y 1998), de establecimiento de la agenda (McCombs, 2006) y de determinación de las tendencias en la opinión pública (Velázquez, 2000; Noelle-Neumann, 1995).

Sin embargo, queremos acotar nuestra reflexión dando cuenta de que si relevamos la importancia que adquiere la prensa, en tanto institución paradigmática y cultural de las sociedades modernas (Thompson, 1998), respecto a la producción de contenidos que determinan los horizontes y límites en los que se enmarcan los mundos posibles (Rodrigo Alsina, 1996; Farré, 2004) en construcción, entonces es posible afirmar que la prensa en su condición de actor del sistema político (Borrat, 1989 y 2003) juega un papel fundamental en la reproducción y legitimación de un sistema de ideas, el cual se sostiene en el proceso de reproducción de imaginarios sociales, proceso donde, además, el discurso de la prensa se encuentra determinado por las condiciones impuestas a las empresas periodísticas que responden a ciertos intereses asociados a marcos hegemónicos articuladores de rutinas y convergencias. Así entonces, considerando lo enunciado, cobra sentido, desde nuestro punto de vista, lo planteado por Manuel Antonio Baeza cuando declara que:

El problema del ideólogo y también del político - a los cuales podría agregar la del publicista y del operador de esas grandes empresas de fabricación de opinión- será siempre aquél de poder promover nuevos imaginarios, o simplemente, manipular imaginarios sociales preexistentes. Ideólogo y político, publicista y periodista juegan en estas maniobras de 'encantamiento' de masas su propia credibilidad, nada más, nada menos. (2008, p. 30).

En otras palabras, el discurso de la prensa se estructura, también, a partir de un conjunto de relatos y valoraciones sobre sucesos que son reconocidos por los sujetos y actores del sistema social en la medida en que toda producción discursiva define los significados que se adosan a hechos sociales articuladores del entramado de la memoria colectiva (Halbwachs, 2004, Mayorga Rojel, Nitrihual y Fierro, 2012) y determinantes de las prácticas culturales vigentes. Por tanto, diremos entonces que el discurso de los medios de comunicación, en general, y de la prensa, en particular, permiten la configuración de relatos y opiniones capaces de acuñar significaciones socioimaginarias (Mayorga, del Valle y Browne, 2013), las cuales, al ser legitimadas institucionalmente, perduran en el tiempo y pasan a transformarse en imaginarios sociales instituidos. Esto quiere decir, según Cornelius Castoriadis (2001) que:

Una vez creadas, tanto las significaciones imaginarias sociales como las instituciones, se cristalizan o se solidifican, y es lo que llamo el imaginario social instituido. Este último asegura la continuidad de la sociedad, la reproducción y la repetición de las mismas formas, que de ahora en más regulan la vida de los hombres y permanecen allí hasta que un cambio histórico lento o una nueva creación masiva venga a modificarlas o a reemplazarlas radicalmente por otras formas (p. 96).

De hecho, si el discurso de la prensa puede ejercer su poder para estructurar un tipo de mundo posible (Rodrigo Alsina, 1996; Farré, 2004) bajo determinadas condiciones 
de consumo establecidas para la apropiación de un contenido que se obtiene mediante una experiencia mediática (Thompson, 1998), entonces tal situación nos permite asumir la existencia de una intencionalidad por parte de la prensa en lo que respecta a lo que puede y debe ser dicho.

En tal caso, el discurso de la prensa posee un valor especial en la reproducción de una ideología determinada (Hall, 1981; Fairclough, 1995; Ferguson, 2007). Esta capacidad que tiene la práctica social del discurso de la prensa facilita la configuración de un campo referencial específico donde los contenidos responden a ciertas significaciones socioimaginarias relacionadas con los intereses de un grupo social (Mayorga, del Valle et al., 2013).

Por consiguiente, podemos decir que el discurso de la prensa, entendida como una práctica social institucionalizada a partir del funcionamiento del sistema de medios de comunicación, corresponde a un agente de socialización que construye realidades y que instituye regímenes de verdad (Foucault, 2009), desde donde es posible construir o reproducir imaginarios sociales.

\section{Propuesta metodológica}

Quisiéramos dar inicio a esta sección del trabajo con un enunciado que orienta la propuesta metodológica de nuestra investigación y que se puede sintetizar de la siguiente forma: existe una capacidad inherente de los medios de comunicación para configurar discursos que facilitan un ordenamiento de la complejidad social en torno a la vida cotidiana sobre la base de un conjunto de imaginarios sociales que se expresan a través de las formas simbólicas acuñadas en los discursos mediáticos.

De este modo, para llevar a cabo esta investigación, se recurrió al análisis del discurso puesto que corresponde a una estrategia teórica y metodológica de carácter interdisciplinaria pertinente para el estudio de la prensa y su producción discursiva en contextos de conflictividad social, la cual se entrelaza en las distintas formaciones sociales para articular el sentido y un tipo de experiencia que adquieren los sujetos en relación con el mundo social.

En este sentido, podemos convenir con Jesús Ibáñez (2003) respecto a que "el orden social es el orden del decir" (p. 64), por tanto, el análisis del discurso de la prensa se justifica en la medida en que todo discurso elaborado por la prensa en contextos de conflicto social suele significar mucho más de lo que literalmente pueda decir y, además, todo discurso es una forma de acción que define el orden social que se establece a través de la práctica discursiva institucional. Además:

El análisis del discurso, en la totalidad de su gama cualitativa, cubre el espectro de todo lo que puede decirse y de las formas en que emerge lo que puede decirse $\mathrm{y}$, por consiguiente, el análisis del discurso puede, por regla general, realizar afirmaciones válidas sobre una o varias facetas del discurso. (Jäger, 2001, p. 69). 
Por consiguiente, en cuanto al análisis del discurso, queda de manifiesto que para los fines de nuestra investigación este tipo de análisis se propone transparentar la opacidad que se conforma mediante el uso del lenguaje en su condición de recurso utilizado por los medios de comunicación para la conformación de la realidad social que se cristaliza sobre la base de elementos simbólicos.

En tal caso, podemos establecer que, con el fin de responder a la pregunta de investigación y al objetivo planteado, se realizó un análisis del discurso en textos periodísticos clasificados en el género de opinión como son las editoriales publicadas por los medios de prensa más relevantes e influyentes de Chile (El Mercurio) y Argentina (La Nación). En términos más precisos, ambos diarios fueron seleccionados según los siguientes criterios:

1. Por la cobertura del territorio nacional (Chile y Argentina).

2. Por la importancia que tienen ambos diarios en la configuración de la opinión pública en sus respectivos territorios de cobertura (Chile y Argentina).

3. Por la antigüedad que poseen ambos diarios en América Latina. En el caso del diario El Mercurio, su primer ejemplar se publicó el 1 de junio de 1900 y en el caso del diario La Nación de Argentina, su primer ejemplar se publicó el 4 de enero de 1870.

4. Por ser ambas empresas periodísticas las fundadoras en 1991 del Grupo de Diarios América (GDA), consorcio mediático que reúne a 11 empresas periodísticas (específicamente del sector de la prensa escrita) que poseen una gran influencia política y económica en sus respectivos mercados nacionales.

Además, para desarrollar la presente investigación centrada en el análisis del imaginario de la protesta social en el discurso editorial de la prensa, se construyó un corpus de textos obtenidos a partir de las editoriales publicadas por el diario El Mercurio y La Nación durante el periodo de tiempo que va desde el 1 diciembre de 2001 al 31 marzo de 2002. La temporalidad definida para la selección de los ejemplares de los diarios se establece con la finalidad de dar cumplimiento a un levantamiento de información pertinente en relación a los criterios de selección del corpus definido en la investigación.

En este sentido, en una primera etapa del trabajo el levantamiento original logra reunir y clasificar una muestra total de 80 editoriales, 36 editoriales fueron publicadas por el diario El Mercurio (Chile) y 44 editoriales fueron publicadas por el diario La Nación (Argentina). En una segunda etapa, se establece la necesidad de seleccionar una submuestra de editoriales para la construcción del corpus de análisis que se utiliza para la obtención de los resultados que se presentan en este trabajo. Así entonces, para la selección de las editoriales que fueron analizadas se utilizaron los criterios que se muestran en la tabla 1. 
La construcción socioimaginaria de la protesta social en el discurso de la prensa latinoamericana: análisis...

Tabla 1. Criterios de selección del corpus textual

\begin{tabular}{|l|l|}
\hline \multicolumn{1}{|c|}{ Criterio } & \multicolumn{1}{c|}{ Descripción del criterio } \\
\hline Tipo de discurso & Todo discurso de la prensa que corresponda a una editorial. \\
\hline $\begin{array}{l}\text { Tópico o temática } \\
\text { central }\end{array}$ & $\begin{array}{l}\text { Todo discurso editorial donde se haga referencia a las acciones colectivas } \\
\text { de protesta y a la crisis argentina de 2001. }\end{array}$ \\
\hline $\begin{array}{l}\text { Tipo de medio de } \\
\text { comunicación }\end{array}$ & $\begin{array}{l}\text { Todo discurso editorial producido por la prensa escrita donde se haga refe- } \\
\text { rencia a las acciones colectivas de protesta y a la crisis argentina de 2001. }\end{array}$ \\
\hline $\begin{array}{l}\text { Identificación de la } \\
\text { prensa escrita }\end{array}$ & $\begin{array}{l}\text { Todo discurso editorial producido por el diario El Mercurio de Chile y La } \\
\text { Nación de Argentina donde se haga referencia a las acciones colectivas de } \\
\text { protesta y a la crisis argentina de 2001. }\end{array}$ \\
\hline Temporalidad & $\begin{array}{l}\text { Todo discurso editorial del diario El Mercurio de Chile y La Nación de } \\
\text { Argentina, publicado entre el 1 de diciembre de 2001 y el 31 de marzo de } \\
2002, \text { y donde se haga referencia a las acciones colectivas de protesta y a } \\
\text { la crisis argentina de 2001. }\end{array}$ \\
\hline
\end{tabular}

Fuente: elaboración propia.

La submuestra seleccionada para realizar el análisis del discurso corresponde a 40 editoriales que fueron publicadas por los diarios El Mercurio (Chile) y La Nación (Argentina). Estas editoriales fueron obtenidas de los ejemplares impresos de cada medio y, además, se utilizó la búsqueda y consulta de las ediciones impresas en formato PDF de cada medio de comunicación a través del buscador del sitio de las respectivas empresas periodísticas.

Tabla 2. Identificación del corpus de análisis

\begin{tabular}{|c|l|l|c|}
\hline N. $^{\circ}$ & \multicolumn{1}{|c|}{ Prensa (Chile) } & \multicolumn{1}{|c|}{ Editoriales } & Fecha de publicación \\
\hline 1 & El Mercurio & Crisis argentina (I) & $09-12-2001$ \\
\hline 2 & El Mercurio & Incertidumbre en Argentina (I) & $15-12-2001$ \\
\hline 3 & El Mercurio & Crisis argentina (II) & $16-12-2001$ \\
\hline 4 & El Mercurio & Incertidumbre en Argentina (II) & $22-12-2001$ \\
\hline 5 & El Mercurio & Frutos de la tercera vía & $26-12-2001$ \\
\hline 6 & El Mercurio & Presidente provisorio en Argentina & $30-12-2001$ \\
\hline 7 & El Mercurio & Efectos de la crisis trasandina & $02-1-2002$ \\
\hline 8 & El Mercurio & Crisis en Argentina & $05-1-2002$ \\
\hline 9 & El Mercurio & $\begin{array}{l}\text { La economía de mercado no es sólo } \\
\text { mercado }\end{array}$ & $06-1-2002$ \\
\hline 10 & El Mercurio & Argentina & $10-1-2002$ \\
\hline 11 & El Mercurio & Incertidumbre en Argentina & $14-1-2002$ \\
\hline
\end{tabular}




\begin{tabular}{|c|c|c|c|}
\hline N. ${ }^{\circ}$ & Prensa (Chile) & Editoriales & Fecha de publicación \\
\hline 12 & El Mercurio & Diagnóstico equivocado & $17-1-2002$ \\
\hline 13 & El Mercurio & Crisis argentina en Chile & $25-1-2002$ \\
\hline 14 & El Mercurio & Argentina: ¿cita con el colapso? & $27-1-2002$ \\
\hline 15 & El Mercurio & Asistencia técnica a Argentina & $30-1-2002$ \\
\hline 16 & El Mercurio & Crisis en aumento & $06-2-2002$ \\
\hline 17 & El Mercurio & Medidas económicas en Argentina & $14-2-2002$ \\
\hline 18 & El Mercurio & La moda liberal & $27-2-2002$ \\
\hline 19 & El Mercurio & Argentina: arresto domiciliario & $05-3-2002$ \\
\hline 20 & El Mercurio & Efectos de la crisis argentina & $09-3-2002$ \\
\hline 1 & La Nación & La crisis y el orden democrático & $08-12-2001$ \\
\hline 2 & La Nación & La reconstrucción de la confianza & $11-12-2001$ \\
\hline 3 & La Nación & Peligros de la provocación disociadora & $13-12-2001$ \\
\hline 4 & La Nación & Quejas que no se pueden desoír & $14-12-2001$ \\
\hline 5 & La Nación & El diálogo es el gran instrumento & $16-12-2001$ \\
\hline 6 & La Nación & Preservar el orden jurídico y social & $18-12-2001$ \\
\hline 7 & La Nación & Ante todo, frenar la violencia & $20-12-2001$ \\
\hline 8 & La Nación & El país necesita una autoridad & $21-12-2001$ \\
\hline 9 & La Nación & La violencia, siempre injustificable & $21-12-2001$ \\
\hline 10 & La Nación & Hacia la restauración del orden & $23-12-2001$ \\
\hline 11 & La Nación & La policía y los hechos de violencia & $23-12-2001$ \\
\hline 12 & La Nación & No equivocar el camino & $27-12-2001$ \\
\hline 13 & La Nación & La función policial y su transparencia & $28-12-2001$ \\
\hline 14 & La Nación & Reconstruir el tejido social & $30-12-2001$ \\
\hline 15 & La Nación & La hora de la unidad nacional & $31-12-2001$ \\
\hline 16 & La Nación & El apego por los cacerolazos & $07-12-2001$ \\
\hline 17 & La Nación & Un llamado a la responsabilidad & $18-12-2001$ \\
\hline 18 & La Nación & Vandalismo y amnistía & $20-12-2001$ \\
\hline 19 & La Nación & Políticos agredidos & $21-12-2001$ \\
\hline 20 & La Nación & Preservar la paz social & $25-12-2001$ \\
\hline
\end{tabular}

Fuente: elaboración propia.

Respecto al modelo de análisis del discurso (véase tabla 3) utilizado en la investigación, debemos mencionar que la aplicación de esta modalidad de análisis fue presentada en el artículo Análisis complejo del discurso: una propuesta metodológica para el estudio de la representación 
mediática en la prensa escrita, que ha sido publicado en la revista científica Anagramas en su edición 13 del año 2008. En el mencionado artículo se detalla el uso del modelo para el análisis de los discursos producidos por la prensa en contextos de conflicto.

Además, este modelo de análisis ha sido aplicado en otras investigaciones (Fondecyt ${ }^{2}$ 1180892; Fondecyt 1150666; Fondecyt 1161253; Diufro 3 DI 12-0038 y Diufro DI18-0047) y publicaciones (Mayorga, Nahuelpi et al., 2013; Mayorga, Del Valle et al., 2013; Mayorga, Nitrihual y Fierro 2012; Mayorga, Del Valle y Nitrihual , 2010; Mayorga y León, 2007) que se han desarrollado en el marco del Programa de Investigación sobre Imaginarios Sociales, Discurso y Poder que se gestiona desde la línea de investigación en Estudios Culturales, Discurso y Comunicación del Doctorado en Ciencias Sociales de la Universidad de La Frontera (Temuco, Chile).

Tabla 3. Componentes del modelo de análisis del discurso

\begin{tabular}{|l|l|}
\hline $\begin{array}{l}\text { Figuras del } \\
\text { discurso }\end{array}$ & $\begin{array}{l}\text { Las figuras son elementos significativos que forman parte de la estructura narrativa } \\
\text { organizadora de todo texto. Son fácilmente reconocibles y se pueden identificar a } \\
\text { partir de la siguiente clasificación: figuras actor, figuras de tiempo y figuras de lugar. }\end{array}$ \\
\hline $\begin{array}{l}\text { Recorridos } \\
\text { figurativos }\end{array}$ & $\begin{array}{l}\text { En los textos, las figuras actor, las figuras de tiempo y las figuras de lugar están } \\
\text { dispuestas en recorridos figurativos que corresponden a la descripción de: 1) las } \\
\text { posiciones y roles que asumen las figuras en el texto y 2) los tipos de relación que se } \\
\text { establecen entre las figuras presentes en el texto en virtud de sus posiciones y roles. }\end{array}$ \\
\hline $\begin{array}{l}\text { Valores } \\
\text { temáticos }\end{array}$ & $\begin{array}{l}\text { Es el significado manifiesto o latente que ostentan las figuras producto de las } \\
\text { posiciones, roles y tipos de relaciones que se establecen entre cada figura que } \\
\text { participa de la estructura narrativa de todo texto. }\end{array}$ \\
\hline $\begin{array}{l}\text { Elementos } \\
\text { nucleares }\end{array}$ & $\begin{array}{l}\text { Todo texto se organiza a partir de una estructura narrativa que se articula sobre } \\
\text { la base de un conjunto de elementos nucleares que corresponden a categorías } \\
\text { y subcategorías significativas capaces de agrupar, ordenar y clasificar los com- } \\
\text { ponentes de un discurso. Así, los elementos nucleares de cada texto analizado } \\
\text { se manifiestan a partir de las relaciones de complementariedad, contradicción y } \\
\text { contrariedad que definen el estatuto lógico de un discurso. }\end{array}$ \\
\hline
\end{tabular}

Fuente: elaboración a partir de las propuestas sobre análisis del discurso formuladas por Giroud y Panier (1988) y Courtés (1995).

El análisis del discurso propuesto se desarrolló en dos fases de continuidad: en una primera instancia se aplicó un análisis de los discursos producidos por los diarios El Mercurio y La Nación considerando, además, un ordenamiento cronológico en lo que respecta a la fecha de publicación de cada editorial. Las orientaciones que se establecieron como guías del análisis corresponden al proceso descriptivo realizado a partir de los tres primeros componentes del modelo de análisis del discurso. Tal decisión se justifica en la medida que se buscaba reconocer el conjunto de figuras del discurso, recorridos figura-

\footnotetext{
Proyectos financiados por el Fondo Nacional de Desarrollo Científico y Tecnológico del Gobierno de Chile.

Proyectos financiados por la Dirección de Investigación de la Universidad de La Frontera (Temuco, Chile).
} 
tivos y valores temáticos presentes en las editoriales que ambos periódicos publicaron en referencia a los sucesos de conflicto social en el contexto de la crisis económica de 2001 en Argentina.

En la segunda instancia de análisis se procede a la interpretación de los resultados obtenidos durante la primera instancia con la finalidad de identificar y describir los elementos nucleares que estructuran el imaginario de la protesta social presentes en las editoriales publicadas por los diarios El Mercurio y La Nación. Así entonces, en esta instancia se procede a construir una representación lógica (véanse figuras 1 y 2) que permite visualizar los elementos nucleares fundamentales que determinan la estructura del imaginario social que subyace en las editoriales analizadas y, por ende, planteamos una modalidad de relato que nos permita destacar los hallazgos más importantes de la investigación en relación con el contexto del estudio.

Por último, en la siguiente sección del texto presentamos solo los principales resultados que se obtuvieron a partir de la segunda instancia de análisis ${ }^{4}$.

\section{Principales resultados: de la civilización y la barbarie a la inclusión y exclusión como elementos nucleares constituyentes del imaginario de la protesta social}

Sobre la base de los resultados obtenidos a partir del análisis del corpus declarado en nuestra investigación, podemos determinar que el diario La Nación construye un imaginario social de la acción colectiva de protesta a partir de dos elementos nucleares presentes en todo el discurso editorial articulado a propósito de los eventos enmarcados en la crisis argentina de 2001, a saber: civilización y barbarie.

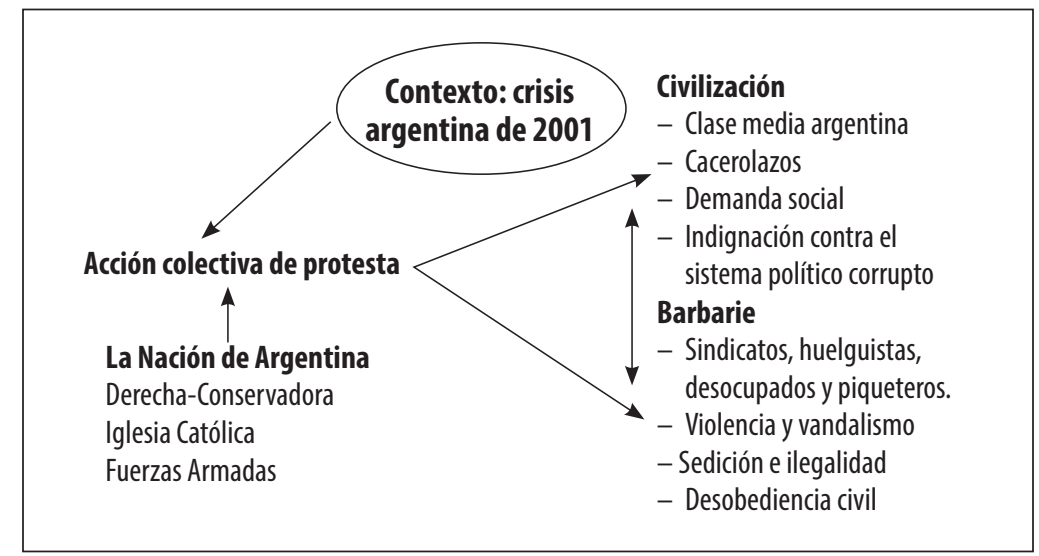

Figura 1. Estructura del imaginario de la protesta social en el discurso editorial del diario La Nación (Argentina)

La información resultante de la primera instancia de análisis no será presentada en detalle en este trabajo, puesto que debemos considerar los requerimientos de extensión definidos por la revista para la publicación de trabajos académicos. 
En efecto, estos elementos nucleares organizan el discurso editorial producido por el diario La Nación respecto a las protestas sociales que se manifiestan en las calles de las ciudades más importantes de la Argentina. Por lo tanto, las huelgas de los trabajadores del sistema público, las marchas y piquetes de los desempleados, los cacerolazos de la clase media, los saqueos y asaltos al comercio, corresponden a las acciones colectivas violentas que son sancionadas por el diario La Nación a partir de la defensa del ordenamiento jurídico y constitucional que existe y prevalece en la Argentina, pero que además son pilares fundamentales para asegurar el derecho a la propiedad y a la libertad de comercio.

En este sentido, el elemento nuclear de la barbarie presente en las editoriales del diario La Nación se articula a partir del significado negativo que se adscribe a las huelgas y acciones colectivas de protesta que son convocadas por las organizaciones sindicales y colectivos sociales organizados en diversas provincias de Argentina, lo que profundiza la división que existe en el país producto de la desconfianza en las capacidades del sistema político argentino para resolver la crisis económica de 2001.

Además, los editoriales publicados por el diario La Nación dan cuenta de que el imaginario de la protesta social se relaciona con la desconfianza que expresa la clase media en el poder político y financiero, y con la sensación de que existe una profunda corrupción en el aparato público que, de una u otra forma, define la naturaleza de la crisis económica del país. Por ende, el elemento nuclear de la barbarie se relaciona en el discurso editorial del diario La Nación con una valoración negativa acerca de la autoridad política que recae en el presidente de la República y su notoria incapacidad para ejercer una gestión político-económica eficiente que permita enfrentar las movilizaciones sociales que se gestan en todas las provincias argentinas.

En tal caso, el discurso editorial del diario La Nación se focaliza en destacar el elemento nuclear de la barbarie a través de una crítica permanente a la ausencia de autoridad en el marco de la grave crisis que afecta a la Argentina. Por tal motivo, este discurso editorial manifiesta que la renuncia de Fernando de la Rúa a la presidencia de la república es una oportunidad para que el pueblo argentino pueda retomar el camino de la democracia y detener la violencia que se ejerce en las calles como único mecanismo social que persigue la imposición de un estado de barbarie que vulnera el respeto al Estado de derecho e impide el funcionamiento, tanto de la economía como de las facultades legislativas del gobierno y del parlamento.

En otras palabras, el análisis del corpus permite evidenciar cómo este discurso editorial relaciona el elemento nuclear de la barbarie con la ausencia de una autoridad y el descontrol de los distintos actores sociales argentinos que promueven acciones delictivas y sediciosas y movilizan al pueblo para participar de los sucesos relacionados con los estallidos sociales y la violencia excesiva que se materializa en las calles.

Sin embargo, también es posible dar cuenta de que el discurso editorial del diario La Nación logra articular el imaginario de la protesta social a partir de un significado positivo en torno a las acciones colectivas que ejecuta la clase media argentina, puesto 
que esta condición de clase adosada a un tipo de sujeto que sufre las consecuencias de la crisis económica no se relaciona con un actuar barbárico, por el contrario, el elemento nuclear de la civilización que se manifiesta en el discurso editorial del diario La Nación se sostiene a partir de una estructura narrativa que destaca a la clase media como un colectivo social capaz de planificar y gestionar diversas manifestaciones, como los apagones, las marchas pacíficas y los cacerolazos, que se llevaron a cabo en el espacio público argentino y que, por lo demás, fueron referenciadas como medidas válidas y legítimas para protestar contra el sistema político corrupto y la intolerable situación de apremio económico que experimentan las personas de clase media producto de la crisis que se vive en el país.

Por consiguiente, es necesario destacar que en el discurso editorial del diario La Nación se refuerza una posición negativa y positiva respecto de la protesta social sobre la base de una comparación que se expresa en el marco de la conflictividad donde, por una parte, existe un tipo de violencia y acciones de vandalismo ejecutadas por sectores populares de la sociedad (piqueteros y sindicalistas) y, por otra, se realizan manifestaciones públicas organizadas por la clase media argentina. Es decir, en el discurso editorial del diario La Nación, los elementos nucleares de civilización y barbarie se expresan mediante el significado positivo y negativo que se adscribe al tipo de acción colectiva de protesta relacionada con la condición de clase que corresponde a la posición social que ocupa el sujeto que protesta en el contexto de la crisis económica de 2001 en Argentina.

El aspecto del análisis que es relevante destacar, corresponde a la capacidad del discurso editorial del diario La Nación para construir un imaginario sobre la protesta social que funciona como un esquema de inteligibilidad donde, por una parte, se advierte que los acontecimientos de vandalismo, violencia, desobediencia civil y descontrol del desorden público serían responsabilidad de un grupo organizado de activistas políticos y sujetos marginales que promueven el caos en el país con la intención de desestabilizar el Estado de derecho y las normas de respeto en torno al orden constitucional y, por otra, se justifica el actuar de las personas de la clase media argentina que se movilizan de forma pacífica y protestan mediante el uso racional de la acción política con el objetivo de expresar su malestar y disconformidad respecto a las medidas implementadas por el gobierno argentino para controlar la crisis económica y la violencia en las calles.

En consecuencia, resulta innegable evidenciar que en el discurso editorial del diario La Nación el imaginario de la protesta social se ha configurado en torno a la centralidad de los elementos nucleares de civilización y barbarie que definen la estructura narrativa de los textos periodísticos analizados. En este sentido, el discurso editorial utiliza los elementos nucleares para relevar de forma positiva la existencia de un tipo de protesta social que se articula a través de acciones colectivas como son los cacerolazos y las manifestaciones públicas que son organizadas por la clase media argentina, en contraste con las formas negativas de la protesta social relacionadas con las huelgas, las marchas, los enfrentamientos con la policía y los saqueos al comercio que son generados por los 
sectores populares, quienes representan la naturaleza violenta de la sociedad argentina e impiden con su actuar organizado el establecimientos de condiciones mínimas para el logro de un acuerdo nacional que proponga respuestas inmediatas a la crisis económica que se experimentaba en Argentina durante el 2001.

Por su parte, el imaginario de la protesta social que se articula en el discurso editorial del diario El Mercurio está relacionado con dos elementos nucleares que estructuran una narrativa centrada en la crisis económica argentina y el modelo de desarrollo económico de libre mercado, a saber: exclusión e inclusión.

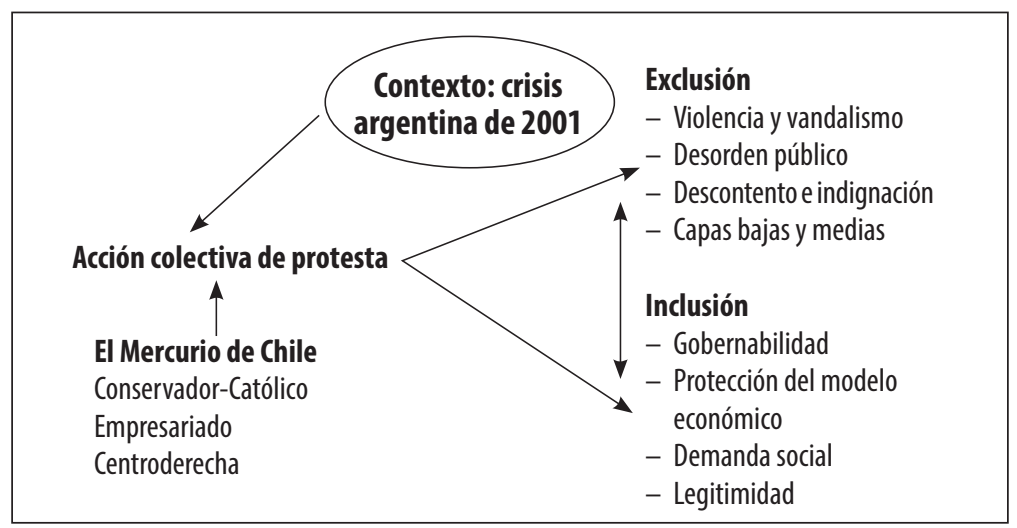

Figura 2. Estructura del imaginario de la protesta social en el discurso editorial del diario El Mercurio (Chile)

Así pues, estos elementos nucleares son centrales en el discurso editorial del diario El Mercurio puesto que, al afirmar que la crisis económica de 2001 se gesta producto de los altos niveles de corrupción en el sistema político y por los errores cometidos en el manejo de la economía de libre mercado, entonces la movilización social de la clase media argentina y el estallido social de la clase popular se justifican como una respuesta, por una parte, a la exclusión de los ciudadanos respecto de los niveles de participación que se requieren para lograr los consensos necesarios en virtud de la resolución de la crisis económica, y, por otra, a la falta de liderazgo y autoridad política para definir el camino que deben tomar las políticas económicas para asegurar la inclusión de la población al desarrollo económico que debe estar sustentado en la estabilidad política del país.

De este modo, el discurso editorial del diario El Mercurio utiliza el elemento nuclear de la exclusión para relevar, por una parte, el aspecto negativo y perjudicial de la crisis económica para la población argentina en el sentido que esta crisis define la relación de conflicto y desconfianza que se establece con las autoridades políticas y, por otra, el sentido positivo de las marchas y cacerolazos que han llevado a cabo las personas de clase media que cuentan con un apoyo transversal de la sociedad civil argentina para posicionar en el espacio público un conjunto de demandas sectoriales relacionadas con los altos niveles de desempleo, mejoras salariales y estabilidad laboral, acceso a 
los ahorros bancarios y control de la violencia callejera que se observa en el marco del conflicto social.

Incluso, en el discurso editorial del diario El Mercurio resulta interesante cómo se logra establecer una diferenciación de sentido que determina la forma como se valoriza y destaca de forma positiva a la protesta social organizada por la clase media argentina en contraposición a la protesta social que moviliza a los sujetos marginales y populares.

Por tanto, la posición de este discurso editorial logra establecer que las demandas enunciadas desde la movilización social articulada por la clase media argentina se transforman en una resistencia al rol interventor del Estado en la economía y expresan los altos niveles de desconfianza que existen en la población con el sistema político. Por el contrario, el discurso editorial del diario El Mercurio destaca la naturaleza anticapitalista de las protestas sociales relacionadas con la clase popular argentina en la medida en que son sus demandas las que apuntan a reforzar la intervención estatal en la economía con la intención de invalidar el funcionamiento del modelo de economía de mercado en el territorio argentino.

Así entonces, a partir del análisis realizado podemos evidenciar cómo el discurso editorial del diario El Mercurio relaciona los elementos nucleares de exclusión e inclusión con una posición de defensa en torno al modelo económico de mercado y la gobernabilidad del territorio. Por tanto, este discurso editorial presenta una estructura narrativa que permite expresar un conjunto de argumentos con la finalidad de evitar una valoración negativa del modelo neoliberal en el contexto de la crisis económica de 2001 en Argentina, lo que se complementa con la posición de los editoriales publicados por el diario El Mercurio respecto a responsabilizar a las autoridades políticas argentinas por la crisis económica y la desconfianza que existe por parte de los ciudadanos y de los organismos financieros globales con las instituciones del país trasandino.

\section{Conclusiones}

A modo de síntesis de nuestro trabajo, creemos necesario destacar que las editoriales publicadas por los diarios El Mercurio y La Nación en el marco del conflicto social que se gesta durante diciembre de 2001 en Argentina, construyeron un imaginario de la protesta social sobre la base de elementos nucleares complementarios que corresponden a los binomios civilización-barbarie y exclusión-inclusión.

De hecho, los discursos editoriales de ambos medios de comunicación funcionaron como esquemas interpretativos que dieron algunas respuestas para comprender la realidad social que experimentaba Argentina durante el 2001 y, por supuesto, orientaron la lectura del conflicto a partir de una estructura narrativa que resaltaba el caos y la violencia en las calles, el deterioro institucional, la pérdida de la confianza en el sistema político, la ausencia de una autoridad presidencial y la profundización de una crisis económicosocial que se produce como consecuencia de los niveles de corrupción que existen en el 
país sumado a los errores cometidos por el gobierno en lo que respecta a la aplicación de medidas proteccionistas y reguladoras de los mercados financieros, lo cual perjudicó el desarrollo de un modelo de economía de libre mercado que se debe sustentar en la autorregulación de los mercados y las libertades individuales de las personas.

Además, los editoriales publicados por los diarios El Mercurio y La Nación reforzaron de forma sistemática un discurso negativo acerca de las protestas sociales organizadas por las clases populares en contraposición a la movilización de la clase media. Al respecto, se logra evidenciar cómo los discursos editoriales de ambos medios determinaron que los problemas de gobernabilidad, violencia y desorden público se explican a partir de las acciones colectivas que llevan a cabo las clases populares. Es decir, desempleados, piqueteros, huelguistas y dirigentes sindicales se convierten en actores sociales negativos para un discurso editorial que, además, visibiliza y relaciona las protestas en las calles, las plazas y los barrios con la desestabilización del gobierno y el quiebre de un orden constitucional que se debe respetar para proteger el derecho a la propiedad privada y la libertad de comercio entre las personas.

En suma, dar cuenta de los resultados obtenidos, producto del análisis de los editoriales publicados por los diarios El Mercurio y La Nación en relación a las movilizaciones que ocurrieron en el contexto de la crisis económica en Argentina, nos permite evidenciar que ambos medios de comunicación jugaron un papel fundamental en lo que respecta a la construcción socioimaginaria de la protesta en ese momento histórico.

Por consiguiente, los diarios El Mercurio y La Nación no solo permitieron visibilizar en el espacio público los acontecimientos asociados a las protestas de diciembre de 2001, sino que, además, estos medios canalizaron, a través de sus discursos editoriales, las explicaciones en torno al conflicto, participaron del debate público respecto a las acciones colectivas de protesta, construyeron representaciones y articularon imaginarios acerca de los sucesos y de los actores sociales que participaron de las interacciones enmarcadas en el conflicto, y, por supuesto, asumieron una posición en su calidad de actores del sistema político respecto al conflicto social que se desarrollaba en la sociedad argentina.

Finalmente, esto implica que debemos entender, entonces, que los diarios El Mercurio y La Nación responden a la categoría de institución mediadora de la realidad puesto que mediante el ejercicio del poder simbólico que ostentan fueron capaces de construir esquemas de interpretación que facilitaron las coordenadas de lectura acerca del conflicto social. En este sentido, estos medios de comunicación, en su calidad de agentes mediadores, poseen la capacidad de construir una realidad sostenida en una arquitectura de significaciones plausibles (Baczko, 2005), por ende, los imaginarios sociales que se cristalizaron a través del discurso editorial de estos medios determinaron el conocimiento en torno a los sucesos ocurridos y ordenaron la vida cotidiana de las audiencias con la finalidad de estructurar toda acción social a partir de la disposición y la legitimidad de sus orientaciones, visiones de mundo, contenidos y opiniones. 


\section{Referencias}

Álvarez, C., Farré, M. y Fernández, D. (2002). Medios de comunicación y protesta social en la crisis argentina: diciembre 2001. Buenos Aires: Konrad Adenauer Stiftung y La Crujía.

Adoni, H y Mane, S. (1984). Media and the social construction of reality. Toward an integration of theory and research. Communication Research, 11(3), 323-340.

Artese, M. (2011). La protesta social y sus representaciones en la prensa argentina entre 1996 y 2002. Perfiles Latinoamericanos, (38), 89-114.

Auyero, J. (2002). Los cambios en el repertorio de la protesta social en Argentina. Desarrollo Económico, 42(166), 187-210.

Auyero, J. (2004). ¿Por qué grita esta gente? Los medios y los significados de la protesta popular en la Argentina de hoy. América Latina Hoy, (36), 161-185.

Auyero, J. (2006). La zona gris. Las características políticas de los saqueos de 2001 en la Argentina. En I. Cheresky (Ed). Ciudadanía, sociedad civil y participación política (pp. 243-270). Buenos Aires: Miño y Dávila Editores.

Auyero, J., y Moran, T. (2007). The Dynamics of Collective Violence: Dissecting Food Riots in Contemporary Argentina. Social Forces, 85(3), 1341 1367.

Baczko, B. (2005). Los imaginarios sociales. Memorias y esperanzas colectivas. Buenos Aires: Nueva Visión.

Baeza, M. (2015). Hacer mundo. Significaciones imaginario-sociales para constituir sociedad. Chile: RIL Editores.

Baeza, M. (2008). Mundo real, mundo imaginario social. Teoría y práctica de sociología profunda. Chile: RIL Editores.

Berger, P. y Luckmann, Th. (2001). La construcción social de la realidad. Buenos Aires: Amorrortu.

Blanks, E. (2008). Black Eye: The Ethics of CBS News and the National Guard Documents. Journal of Mass Media Ethics, 23(2), 90-109.

Borrat, H. (1989). El periódico, actor del sistema político. Revista Análisi: Quaderns de Comunicació i Cultura, (12), 67-80.

Borrat, H. (2003). Narradores en interacción. Revista Científica de Información y Comunicación, (1), 59-84.

Brym, R., Godbout, M., Hoffbauer, A., Menard, G. y Zhang, T. H. (2014). Social media in the 2011 Egyptian uprising. The British Journal of Sociology, 65(2), 266-292.

Carter, M. (2013). The Hermeneutics of Frames and Framing: An Examination of the Media's Construction of Reality. Sage Open, 3, 1-12.

Castoriadis, C. (2001). Figuras de lo pensable. Las encrucijadas del laberinto VI. México: Fondo de Cultura Económica.

Courtés, J. (1995). Análisis semiótico del discurso: del enunciado a la enunciación. Madrid: Gredos.

Curran, J. (2002). Medios de comunicación y poder en una sociedad democrática. Barcelona: Hacer.

Farré, M. (2004). El noticiero como mundo posible. Estrategias ficcionales. Buenos Aires: La Crujía.

Ferrero, J. P. (2017). Post-neoliberal protest in Latin America as a struggle over the name of 'the people'. Journal of Political Ideologices, 22(1), 52-73. 
Ferguson, R. (2007). Los medios bajo sospecha. Ideología y poder en los medios de comunicación. Barcelona: Gedisa.

Foucault, M. (2009). La arqueología del saber. Madrid: Siglo XXI

Giroud, J. C. y Panier, L. (1988). Semiótica. Navarra: Verbo Divino.

Gronemeyer, M. E. y Porath, W. (2017). Tendencias de la posición editorial en diarios de referencia en Chile. El arte de dosificar la crítica frente a la actuación de los actores políticos. Revista de Ciencia Política, 37(1), 177-202.

Halbwachs, M. (2004). La memoria colectiva. Zaragoza: Prensas Universitarias de Zaragoza.

Hall, S. (1981). La cultura, los medios de comunicación y el efecto ideológico. En J. Curran, M. Gurevitch y J. Woollacott (Eds.), Sociedad y comunicación de masas (pp. 221-254). Ciudad de México: Fondo de Cultura Económica.

Hall, S. (1998). Significado, representación e ideología: Althusser y los debates postestructuralistas. En J. Curran, D. Morley y V. Walkerdine (Eds.), Estudios culturales y comunicación. Análisis, producción y consumo de las políticas de la identidad y el posmodernismo (pp. 193-220). Barcelona: Paidós.

Ibáñez, J. (2003). Perspectivas de la investigación social: el diseño en las tres perspectivas. En M. García, J. Ibáñez y F. Alvira (Eds.), El análisis de la realidad social: métodos y técnicas de investigación (pp. 42-75). Madrid: Alianza.

Iñigo Carrera, N. y Cotarelo, M. (2006). Génesis y desarrollo de la insurrección espontánea de diciembre de 2001 en Argentina. En G. Caetano (Ed.), Sujetos sociales y nuevas formas de protesta en la historia reciente de América Latina (pp. 49-92). Buenos Aires: CLACSO.

Jäger, S. (2001). Discurso y conocimiento: aspectos teóricos y metodológicos de la crítica del discurso y del análisis de dispositivos. En R. Wodak y M. Meyer (Eds.), Métodos de análisis crítico del discurso (pp.61-99). Barcelona: Gedisa.

Lukin, A. (2012). Journalism, ideology and linguistics. The paradox of Chomsky's linguistic legacy and his propaganda model. Journalism, 14(1). 96-110.

Lunt, P. y Livingstone, S. (2013). Media studies fascination with the concept of the public sphere: critical reflections and emerging debates. Media, Culture and Society, 35(1), 87-96.

Martín-Barbero, J. (2004). Oficio de cartógrafo. Travesías latinoamericanas de la comunicación en la cultura. Argentina: Fondo de Cultura Económica.

Mastrini, G. y Becerra, M. (2006). Periodistas y magnates. Estructura y concentración de las industrias culturales en América Latina. Buenos Aires: Prometeo e IPyS.

Mauro, S. y Natanson, J. (2006). Ciudadanía y sociedad civil en la ciudad de Buenos Aires. El espacio público entre la audiencia y el estallido. En I. Cheresky (Ed.), Ciudadanía, sociedad civil y participación política (pp.217-248). Buenos Aires: Miño y Dávila Editores.

Mayorga Rojel, A. y León, C. (2007). El malvado siempre es el otro: Perú y su construcción discursiva en la prensa chilena. Revista Universum, 22(2), 164-176.

Mayorga Rojel, A., Del Valle, C. y Nitrihual, L. (2008). Análisis complejo del discurso. Una propuesta metodológica para el estudio de la representación mediática en la prensa escrita. Anagramas Rumbos y Sentidos de la Comunicación, 7(13), 13-33. 
Mayorga Rojel, A., Del Valle, C. y Nitrihual, L. (2010). Entre memoria y conflicto. La construcción mediática de la otredad en el marco del conflicto limítrofe entre Chile y Perú. Revista Comunicación y Medios, (21), 51-71.

Mayorga Rojel, A., Nitrihual, L. y Fierro, J. (2012). Imaginario social, memoria colectiva y construcción de territorios en torno a los 30 años del golpe militar en Chile. Anagramas Rumbos y Sentidos de la Comunicación, 10(20), 19-36.

Mayorga Rojel, A., Nahuelpi, C. y Nitrihual, L. (2013). El imaginario social de la mujer mapuche en el discurso de la prensa en Chile. El ejemplo del diario El Austral de La Araucanía. Revista Estudios sobre el Mensaje Periodístico, 19(2), 767-782.

Mayorga Rojel, A., Del Valle, C. y Browne, R. (2013). El imaginario social de la acción colectiva de protesta y la crisis argentina de 2001 en el discurso de la prensa en Chile. Revista Polis, 12(34), 1-20.

McCombs, M. (2006). Estableciendo la agenda: el impacto de los medios en la opinión pública y en el conocimiento. Barcelona: Paidós.

Moreno Sardá, A., Simelio Solá, N., Rovetto, F. y Buitrago, A. (2007). Periodismo y ciudadanía plural: problemas, rutinas y retos. Revista Estudios sobre el Mensaje Periodístico, 13, 157-168.

Raimondo Anselmino, N. (2014). Prensa online y tipos de lectores: respuestas del público y lógicas de reconocimiento en los comentarios a las noticias del diario argentino La Nación. Cuadernos. info, (34), 183-195.

Neuhaus, S. (2002). Discurso hegemónico en la des-construcción del espacio público y la subjetividad. Buenos Aires: Altamira.

Noelle-Neumann, E. (1995). La espiral del silencio. Opinión pública: nuestra piel social. Barcelona: Paidós.

Rodrigo Alsina, M. (1996). La construcción de la noticia. Barcelona: Paidós.

Rodríguez, M. (2006). Representación política, deliberación y acción colectiva. Piqueteros y asambleísta: dos figuras de la participación de la sociedad civil en Argentina. En I. Cheresky (Ed.), Ciudadanía, sociedad civil y participación política (pp. 317-344). Buenos Aires: Miño y Dávila Editores.

Rossi, F y Mauro, S. (2013). The Movement of Popular and Neighborhood Assemblies in the City of Buenos Aires, 2002-2011. Latin American Perspectives, 42(2), 107-124.

Rossi, F. (2017). The Poor's Struggle for Political Incorporation. The Piquetero Movement in Argentina. Londres: Cambridge University Press.

Simelio, N. (2009). Información y género: la representación de las mujeres en la prensa. Diagnóstico y propuestas. En S. Alcoba. (Coord.), Lengua, comunicación y libros de estilo (pp. 323-335). Barcelona: Universidad Autónoma de Barcelona.

Sodré, M. (1998). Reinventado la cultura. La comunicación y sus productos. Barcelona: Gedisa

Thompson, J. B. (1998). Los media y la modernidad. Una teoría de los medios de comunicación. Barcelona: Paidós

Velázquez, T. (1992). Realidad trópica y discurso audiovisual. En G. Deledalle, M. Balat y J. DeledalleRhodes (Eds.), Signs of Humanity (Vol III.) (pp. 1683-1692). Berlín: Mouton de Gruyter.

Velázquez, T. (2000). Televisión y elecciones presidenciales 2000. Revista Mexicana de Ciencias Política y Sociales, 44(180), 65-88. 